EUROPEAN LABORATORY FOR PARTICLE PHYSICS (CERN)

CERN-EP/99-092

8 July 1999

\title{
Determination of the LEP centre-of-mass energy from $Z \gamma$ events
}

\author{
The ALEPH Collaboration
}

\begin{abstract}
Radiative returns to the $Z$ resonance ( $Z \gamma$ events) are used to determine the LEP2 centreof-mass energy from the data collected with the ALEPH detector in 1997. The average centre-of-mass energy is measured to be:

$$
E_{C M}=182.50 \pm 0.19 \text { (stat.) } \pm 0.08 \text { (syst.) } \mathrm{GeV}
$$

in good agreement with the precise determination by the LEP energy working group of $182.652 \pm 0.050 \mathrm{GeV}$. If applied to the measurement of the $W$ mass, its precision translates into a systematic error on $M_{W}$ which is smaller than the statistical error achieved from the corresponding dataset.
\end{abstract}

Submitted to Physics Letters B 


\section{The ALEPH Collaboration}

R. Barate, D. Decamp, P. Ghez, C. Goy, S. Jezequel, J.-P. Lees, F. Martin, E. Merle, M.-N. Minard, B. Pietrzyk, H. Przysiezniak

Laboratoire de Physique des Particules (LAPP), IN $N^{2} P^{3}$-CNRS, F-74019 Annecy-le-Vieux Cedex, France

R. Alemany, M.P. Casado, M. Chmeissani, J.M. Crespo, E. Fernandez, M. Fernandez-Bosman, Ll. Garrido, ${ }^{15}$ E. Graugès, A. Juste, M. Martinez, G. Merino, R. Miquel, Ll.M. Mir, P. Morawitz, A. Pacheco, I.C. Park, I. Riu

Institut de Física d'Altes Energies, Universitat Autònoma de Barcelona, 08193 Bellaterra (Barcelona), E-Spain ${ }^{7}$

A. Colaleo, D. Creanza, M. de Palma, G. Iaselli, G. Maggi, M. Maggi, S. Nuzzo, A. Ranieri, G. Raso, F. Ruggieri, G. Selvaggi, L. Silvestris, P. Tempesta, A. Tricomi, ${ }^{3}$ G. Zito

Dipartimento di Fisica, INFN Sezione di Bari, I-70126 Bari, Italy

X. Huang, J. Lin, Q. Ouyang, T. Wang, Y. Xie, R. Xu, S. Xue, J. Zhang, L. Zhang, W. Zhao

Institute of High-Energy Physics, Academia Sinica, Beijing, The People's Republic of China ${ }^{8}$

D. Abbaneo, U. Becker, ${ }^{19}$ G. Boix,${ }^{6}$ M. Cattaneo, F. Cerutti, V. Ciulli, G. Dissertori, H. Drevermann, R.W. Forty, M. Frank, F. Gianotti, T.C. Greening, A.W. Halley, J.B. Hansen, J. Harvey, P. Janot, B. Jost, I. Lehraus, O. Leroy, C. Loomis, P. Maley, P. Mato, A. Minten, A. Moutoussi, F. Ranjard, L. Rolandi, D. Schlatter, M. Schmitt, ${ }^{20}$ O. Schneider, ${ }^{2}$ P. Spagnolo, W. Tejessy, F. Teubert, I.R. Tomalin, E. Tournefier, A.E. Wright

European Laboratory for Particle Physics (CERN), CH-1211 Geneva 23, Switzerland

Z. Ajaltouni, F. Badaud G. Chazelle, O. Deschamps, S. Dessagne, A. Falvard, C. Ferdi, P. Gay, C. Guicheney, P. Henrard, J. Jousset, B. Michel, S. Monteil, J-C. Montret, D. Pallin, P. Perret, F. Podlyski

Laboratoire de Physique Corpusculaire, Université Blaise Pascal, $I N^{2} P^{3}$-CNRS, Clermont-Ferrand, F-63177 Aubière, France

J.D. Hansen, J.R. Hansen, P.H. Hansen, B.S. Nilsson, B. Rensch, A. Wäänänen

Niels Bohr Institute, 2100 Copenhagen, DK-Denmark ${ }^{9}$

G. Daskalakis, A. Kyriakis, C. Markou, E. Simopoulou, A. Vayaki

Nuclear Research Center Demokritos (NRCD), GR-15310 Attiki, Greece

A. Blondel, J.-C. Brient, F. Machefert, A. Rougé, M. Swynghedauw, R. Tanaka, A. Valassi, ${ }^{23}$ H. Videau

Laboratoire de Physique Nucléaire et des Hautes Energies, Ecole Polytechnique, IN $\mathrm{IN}^{2} \mathrm{P}^{3}$ CNRS, F-91128 Palaiseau Cedex, France

E. Focardi, G. Parrini, K. Zachariadou

Dipartimento di Fisica, Università di Firenze, INFN Sezione di Firenze, I-50125 Firenze, Italy

R. Cavanaugh, M. Corden, C. Georgiopoulos

Supercomputer Computations Research Institute, Florida State University, Tallahassee, FL 323064052, USA ${ }^{13,14}$

A. Antonelli, G. Bencivenni, G. Bologna, ${ }^{4}$ F. Bossi, P. Campana, G. Capon, V. Chiarella, P. Laurelli, G. Mannocchi, ${ }^{1,5}$ F. Murtas, G.P. Murtas, L. Passalacqua, M. Pepe-Altarelli ${ }^{1}$

Laboratori Nazionali dell'INFN (LNF-INFN), I-00044 Frascati, Italy

M. Chalmers, L. Curtis, J.G. Lynch, P. Negus, V. O’Shea, B. Raeven, C. Raine, D. Smith, P. TeixeiraDias, A.S. Thompson, J.J. Ward

Department of Physics and Astronomy, University of Glasgow, Glasgow G12 8QQ,United Kingdom ${ }^{10}$ 
O. Buchmüller, S. Dhamotharan, C. Geweniger, P. Hanke, G. Hansper, V. Hepp, E.E. Kluge, A. Putzer, J. Sommer, K. Tittel, S. Werner, ${ }^{19}$ M. Wunsch

Institut für Hochenergiephysik, Universität Heidelberg, D-69120 Heidelberg, Germany ${ }^{16}$

R. Beuselinck, D.M. Binnie, W. Cameron, P.J. Dornan, ${ }^{1}$ M. Girone, S. Goodsir, N. Marinelli, E.B. Martin, J. Nash, J. Nowell, A. Sciabà, J.K. Sedgbeer, E. Thomson, M.D. Williams

Department of Physics, Imperial College, London SW7 2BZ, United Kingdom ${ }^{10}$

V.M. Ghete, P. Girtler, E. Kneringer, D. Kuhn, G. Rudolph

Institut für Experimentalphysik, Universität Innsbruck, A-6020 Innsbruck, Austria ${ }^{18}$

C.K. Bowdery, P.G. Buck, G. Ellis, A.J. Finch, F. Foster, G. Hughes, R.W.L. Jones, N.A. Robertson, M. Smizanska, M.I. Williams

Department of Physics, University of Lancaster, Lancaster LA1 4YB, United Kingdom ${ }^{10}$

I. Giehl, F. Hölldorfer, K. Jakobs, K. Kleinknecht, M. Kröcker, A.-S. Müller, H.-A. Nürnberger, G. Quast, B. Renk, E. Rohne, H.-G. Sander, S. Schmeling, H. Wachsmuth C. Zeitnitz, T. Ziegler

Institut für Physik, Universität Mainz, D-55099 Mainz, Germany ${ }^{16}$

J.J. Aubert, C. Benchouk, A. Bonissent, J. Carr, ${ }^{1}$ P. Coyle, A. Ealet, D. Fouchez, F. Motsch, P. Payre, D. Rousseau, M. Talby, M. Thulasidas, A. Tilquin

Centre de Physique des Particules, Faculté des Sciences de Luminy, IN ${ }^{2} P^{3}$-CNRS, F-13288 Marseille, France

M. Aleppo, M. Antonelli, S. Gilardoni, F. Ragusa

Dipartimento di Fisica, Università di Milano e INFN Sezione di Milano, I-20133 Milano, Italy.

V. Büscher, H. Dietl, G. Ganis, K. Hüttmann, G. Lütjens, C. Mannert, W. Männer, H.-G. Moser, S. Schael, R. Settles, H. Seywerd, H. Stenzel, W. Wiedenmann, G. Wolf

Max-Planck-Institut für Physik, Werner-Heisenberg-Institut, D-80805 München, Germany

P. Azzurri, J. Boucrot, O. Callot, S. Chen, M. Davier, L. Duflot, J.-F. Grivaz, Ph. Heusse, A. Jacholkowska, ${ }^{1}$ M. Kado, J. Lefrançois, L. Serin, J.-J. Veillet, I. Videau, ${ }^{1}$ J.-B. de Vivie de Régie, D. Zerwas

Laboratoire de l'Accélérateur Linéaire, Université de Paris-Sud, IN ${ }^{2} P^{3}$-CNRS, F-91898 Orsay Cedex, France

G. Bagliesi, S. Bettarini, T. Boccali, C. Bozzi ${ }^{12}$ G. Calderini, R. Dell'Orso, I. Ferrante, A. Giassi, A. Gregorio, F. Ligabue, A. Lusiani, P.S. Marrocchesi, A. Messineo, F. Palla, G. Rizzo, G. Sanguinetti, G. Sguazzoni, R. Tenchini, C. Vannini, A. Venturi, P.G. Verdini

Dipartimento di Fisica dell'Università, INFN Sezione di Pisa, e Scuola Normale Superiore, I-56010 Pisa, Italy

G.A. Blair, J. Coles, G. Cowan, M.G. Green, D.E. Hutchcroft, L.T. Jones, T. Medcalf, J.A. Strong, J.H. von Wimmersperg-Toeller

Department of Physics, Royal Holloway \& Bedford New College, University of London, Surrey TW20 OEX, United Kingdom ${ }^{10}$

D.R. Botterill, R.W. Clifft, T.R. Edgecock, P.R. Norton, J.C. Thompson Particle Physics Dept., Rutherford Appleton Laboratory, Chilton, Didcot, Oxon OX11 OQX, United Kingdom ${ }^{10}$

B. Bloch-Devaux, P. Colas, B. Fabbro, G. Faï, E. Lançon, M.-C. Lemaire, E. Locci, P. Perez, J. Rander, J.-F. Renardy, A. Rosowsky, A. Trabelsi, ${ }^{21}$ B. Tuchming, B. Vallage

CEA, DAPNIA/Service de Physique des Particules, CE-Saclay, F-91191 Gif-sur-Yvette Cedex, France $^{17}$

S.N. Black, J.H. Dann, H.Y. Kim, N. Konstantinidis, A.M. Litke, M.A. McNeil, G. Taylor

Institute for Particle Physics, University of California at Santa Cruz, Santa Cruz, CA 95064, USA ${ }^{22}$

C.N. Booth, S. Cartwright, F. Combley, P.N. Hodgson, M.S. Kelly, M. Lehto, L.F. Thompson

Department of Physics, University of Sheffield, Sheffield S3 7RH, United Kingdom ${ }^{10}$ 
K. Affholderbach, A. Böhrer, S. Brandt, C. Grupen, J. Hess, A. Misiejuk, G. Prange, U. Sieler

Fachbereich Physik, Universität Siegen, D-57068 Siegen, Germany ${ }^{16}$

G. Giannini, B. Gobbo

Dipartimento di Fisica, Università di Trieste e INFN Sezione di Trieste, I-34127 Trieste, Italy

J. Putz, J. Rothberg, S. Wasserbaech, R.W. Williams

Experimental Elementary Particle Physics, University of Washington, WA 98195 Seattle, U.S.A.

S.R. Armstrong, P. Elmer, D.P.S. Ferguson, Y. Gao, S. González, O.J. Hayes, H. Hu, S. Jin, P.A. McNamara III, J. Nielsen, W. Orejudos, Y.B. Pan, Y. Saadi, I.J. Scott, J. Walsh, Sau Lan Wu, X. Wu, G. Zobernig

Department of Physics, University of Wisconsin, Madison, WI 53706, USA ${ }^{11}$

\footnotetext{
${ }^{1}$ Also at CERN, 1211 Geneva 23, Switzerland.

${ }^{2}$ Now at Université de Lausanne, 1015 Lausanne, Switzerland.

${ }^{3}$ Also at Centro Siciliano di Fisica Nucleare e Struttura della Materia, INFN Sezione di Catania, 95129 Catania, Italy.

${ }^{4}$ Also Istituto di Fisica Generale, Università di Torino, 10125 Torino, Italy.

${ }^{5}$ Also Istituto di Cosmo-Geofisica del C.N.R., Torino, Italy.

${ }^{6}$ Supported by the Commission of the European Communities, contract ERBFMBICT982894.

${ }^{7}$ Supported by CICYT, Spain.

${ }^{8}$ Supported by the National Science Foundation of China.

${ }^{9}$ Supported by the Danish Natural Science Research Council.

${ }^{10}$ Supported by the UK Particle Physics and Astronomy Research Council.

${ }^{11}$ Supported by the US Department of Energy, grant DE-FG0295-ER40896.

${ }^{12}$ Now at INFN Sezione di Ferrara, 44100 Ferrara, Italy.

${ }^{13}$ Supported by the US Department of Energy, contract DE-FG05-92ER40742.

${ }^{14}$ Supported by the US Department of Energy, contract DE-FC05-85ER250000.

${ }^{15}$ Permanent address: Universitat de Barcelona, 08208 Barcelona, Spain.

${ }^{16}$ Supported by the Bundesministerium für Bildung, Wissenschaft, Forschung und Technologie, Germany.

${ }^{17}$ Supported by the Direction des Sciences de la Matière, C.E.A.

${ }^{18}$ Supported by Fonds zur Förderung der wissenschaftlichen Forschung, Austria.

${ }^{19}$ Now at SAP AG, 69185 Walldorf, Germany

${ }^{20}$ Now at Harvard University, Cambridge, MA 02138, U.S.A.

${ }^{21}$ Now at Département de Physique, Faculté des Sciences de Tunis, 1060 Le Belvédère, Tunisia.

${ }^{22}$ Supported by the US Department of Energy, grant DE-FG03-92ER40689.

${ }^{23}$ Now at LAL, 91898 Orsay, France.
} 


\section{Introduction}

One of the main goals of LEP2 is the direct measurement of the $W$ mass with an accuracy better than $30 \mathrm{MeV}$. This requires a very precise determination of the LEP centreof-mass energy, in order to minimize its contribution to the $W$ mass uncertainty. At LEP1 this accuracy was achieved using the Resonant Depolarisation method for energy measurement [1]. At LEP2 this is no longer possible since polarization has not been achieved above a beam energy of $60 \mathrm{GeV}$. Therefore, the precise Resonant Depolarisation calibration is performed only in the $40-55 \mathrm{GeV}$ energy range, and extrapolation to higher energies is necessary. The precision achieved on the $183 \mathrm{GeV}$ centre-of-mass energy range is about $\pm 50 \mathrm{MeV}$ [2]. For future data taking there is hope to improve this precision using the recently installed magnetic spectrometer [3].

An alternative determination is considered in this paper. It is based on an analysis of $e^{+} e^{-} \rightarrow \gamma f \bar{f}$ events, where the Initial State Radiation (ISR) photons are mostly collinear to the beam pipe and remain undetected. In these events, the invariant mass, $\sqrt{s^{\prime}}$, of the final state $f \bar{f}$ system peaks naturally at the $Z$ mass. The variable $x=1-s^{\prime} / s$ can be reconstructed from the final state particle angles which are well measured quantities. Since the $Z$ mass is very well known from LEP1, a fit to the distribution of $x$ can be used to determine the center-of-mass energy $\sqrt{s}$.

This letter presents an application of this method using the $q \bar{q}$ final state in data recorded by ALEPH in 1997. LEP ran at nominal centre-of-mass energies of 181, 182, 183 and $184 \mathrm{GeV}$, and an integrated luminosity of $56.812 \mathrm{pb}^{-1}$ was collected.

\section{The ALEPH detector}

A detailed description of the ALEPH detector can be found in Ref. [5] and of its performance in Ref. [6]. Charged particles are detected in the central part of the detector. From the beam crossing point outwards, a silicon vertex detector, a cylindrical drift chamber, and a large time projection chamber (TPC) measure up to 31 coordinates along the charged particle trajectories. A $1.5 \mathrm{~T}$ axial magnetic field is provided by a superconducting solenoidal coil. A resolution of $\delta p_{T} / p_{T}=6 \times 10^{-4} p_{T} \oplus 0.005\left(p_{T}\right.$ in $\mathrm{GeV} / c)$ is achieved. Hereafter, charged particle tracks reconstructed from at least four hits in the TPC, having a polar angle to the beam axis satisfying $|\cos \theta|<0.95$, and originating from within a cylinder of $2 \mathrm{~cm}$ radius and $20 \mathrm{~cm}$ length, centred on the nominal interaction point and parallel to the beam axis, are called good tracks.

Electrons and photons are identified in the electromagnetic calorimeter by their shower profile. The calorimeter, a lead/wire-plane sampling device with fine readout segmentation and total thickness of 22 radiation lengths at normal incidence, provides an energy resolution $\Delta E / E$ of $0.180 / \sqrt{E}+0.009(E$ in $\mathrm{GeV})$.

Muons are identified by their penetration pattern in the hadron calorimeter, a $1.2 \mathrm{~m}$ thick iron yoke instrumented with 23 layers of streamer tubes, together with two surrounding layers of muon chambers. In association with the electromagnetic calorimeter, the hadron calorimeter also provides a measurement of the energy of charged and neutral hadrons with a relative resolution of $0.85 / \sqrt{E}(E$ in $\mathrm{GeV})$. 
The total visible energy and momentum, as well as the missing energy, are evaluated by an energy flow reconstruction algorithm [6] which combines all of the above measurements, complemented at low polar angles by the energy detected in the luminosity calorimeters. Jets are built from charged and neutral objects reconstructed by the energy flow algorithm. The typical jet angular resolution is $30 \mathrm{mrad}$. The jet energy resolution is approximately $\sigma_{E}=(0.6 \sqrt{E}+0.6) \mathrm{GeV} \cdot\left(1+\cos ^{2} \theta\right)$, where $E$ (in $\mathrm{GeV}$ ) and $\theta$ are the jet energy and polar angle. The jet energy and angular resolution as well as calibrations were obtained from extensive studies of $Z \rightarrow q \bar{q}$ events both in data and Monte Carlo. Discrepancies between data and simulation were used when evaluating systematic errors.

\section{Monte Carlo samples}

A sample of 200,000 $q \bar{q}$ events was generated using KORALZ v4.2 [7] at a nominal energy of $182.675 \mathrm{GeV}$ and fully simulated in the detector. Monte Carlo samples at $183 \mathrm{GeV}$ with integrated luminosities at least 20 times larger than recorded were simulated for all background reactions. PYTHIA v5.7 [8] was used to generate $Z Z$ and Zee events, and KORALW v1.21 [9] to produce four fermion events with $W W$ topologies. Two-photon $(\gamma \gamma)$ reactions into hadrons were simulated with PHOT02 [11] and PHOJET [12]. The method was calibrated with fully simulated $q \bar{q}$ samples of 20,000 events, generated with KORALZ, at 181, 182, 184 and $185 \mathrm{GeV}$.

\section{Event selection and reconstruction algorithm}

At $\sqrt{s}=183 \mathrm{GeV}$ the main backgrounds to the process $e^{+} e^{-} \rightarrow Z \gamma \rightarrow q \bar{q} \gamma$ are $e^{+} e^{-} \rightarrow W W, e^{+} e^{-} \rightarrow Z Z, e^{+} e^{-} \rightarrow \gamma \gamma$ and $e^{+} e^{-} \rightarrow Z e e$. To discriminate between signal and background events, the following cuts are applied.

- $q \bar{q}$ candidates are required to have at least seven good tracks which total energy exceeds $10 \%$ of the nominal centre-of-mass energy.

- The energy flow objects are clustered into jets using the JADE algorithm 113 with a $y_{c u t}$ value of 0.008. Events containing jets with an electromagnetic content of at least $90 \%$ and an energy larger than $10 \mathrm{GeV}$ are rejected.

- The total visible invariant mass is required to be greater than $50 \mathrm{GeV} / c^{2}$.

- The remaining events are then forced into two jets using the DURHAM-E algorithm. The polar angle of both jets, $\theta$, is restricted to $|\cos \theta|<0.95$.

To reconstruct the effective centre-of-mass energy $\sqrt{s^{\prime}}$, it is assumed that the ISR photon is emitted along the beam pipe, resulting in a boost of the $f \bar{f}$ system, or the produced $Z$, in the opposite direction. A kinematic reconstruction based on rescaling of the jet energies is performed for each event. According to Monte Carlo studies, jet velocities and angles are well measured. Each jet four-momentum is scaled by a factor $\alpha$ according to the relations 


$$
\begin{aligned}
& E_{1}^{r e c}=\alpha_{1} E_{1}^{\text {meas }} \quad ; \quad{\overrightarrow{P_{1}^{r e c}}}^{r e \alpha_{1}}{\overrightarrow{P_{1}}}^{\text {meas }} \\
& E_{2}^{r e c}=\alpha_{2} E_{2}^{\text {meas }} \quad ; \quad{\overrightarrow{P_{2}}}^{r e c}=\alpha_{2}{\overrightarrow{P_{2}}}^{\text {meas }}
\end{aligned}
$$

where rec stands for reconstructed and meas for measured. Correction coefficients are different for the two jets.

In the absence of final state radiation (FSR), the correction coefficients $\alpha_{1}$ and $\alpha_{2}$ are obtained by applying energy and momentum conservation, and the assumption that one zero mass particle escapes detection (the ISR $\gamma$ ) along the beam axis. The solution kept is the one which yields $\alpha_{1}$ and $\alpha_{2}$ positive and closest to 1 . Then, the effective centre-of-mass energy can be expressed as

$$
s^{\prime}=s F\left(\frac{\vec{P}_{1}^{\text {meas }}}{E_{1}^{\text {meas }}}, \frac{\vec{P}_{2}^{\text {meas }}}{E_{2}^{\text {meas }}}\right)
$$

from which the quantity $x=1-\left(s^{\prime} / s\right)$ is reconstructed independently of the nominal centre-of-mass energy.

The fit to the centre-of-mass energy is performed for events with $0.60<x<0.88$ where the highest purity and the maximum sensitivity is achieved. The generated cross-sections,

\begin{tabular}{|c|c|c|c|}
\hline Processes & $\sigma(\mathrm{pb})$ & $\sigma_{e f f}(\mathrm{pb})$ & $\sigma_{\text {window }}(\mathrm{pb})$ \\
\hline$q \bar{q} \gamma$ & $108.84 \pm 0.08$ & $68.61 \pm 0.20$ & $40.07 \pm 0.15$ \\
\hline$\overline{W W}$ & $16.02 \pm 0.01$ & $10.51 \pm 0.05$ & $0.47 \pm 0.01$ \\
\hline$Z Z$ & $2.545 \pm 0.13$ & $1.08 \pm 0.05$ & $0.33 \pm 0.02$ \\
\hline$\gamma \gamma \rightarrow$ hadrons (untagged) & 7800. \pm 390 & $0.98 \pm 0.22$ & $0.24 \pm 0.11$ \\
\hline$\gamma \gamma \rightarrow u / d$ & $474.00 \pm 23.70$ & $0.61 \pm 0.08$ & $0.19 \pm 0.04$ \\
\hline$\gamma \gamma \rightarrow s s$ & $26.00 \pm 1.30$ & $0.04 \pm 0.02$ & $0.01 \pm 0.01$ \\
\hline$\gamma \gamma \rightarrow c c$ & $93.60 \pm 4.68$ & $0.55 \pm 0.008$ & $0.19 \pm 0.05$ \\
\hline$\gamma \gamma \rightarrow b b$ & $0.53 \pm 0.03$ & $0.02 \pm 0.005$ & $0.01 \pm 0.01$ \\
\hline$Z e e$ & $6.80 \pm 0.27$ & $4.57 \pm 0.18$ & $1.02 \pm 0.04$ \\
\hline$W e \nu$ & $0.608 \pm 0.03$ & $0.32 \pm 0.02$ & $0.11 \pm 0.01$ \\
\hline \multicolumn{2}{|l|}{ Purity (\%) } & $78.60 \pm 0.01$ & $93.93 \pm 0.01$ \\
\hline
\end{tabular}
the effective ones after selection cuts and within the fitting window are summarised for each process in Table 1 .

Table 1: Generated (second column) and effective cross-section after selection cuts (third column) and within the fitting window (fourth column), for signal and background processes (first column) at $\sqrt{s}=182.675$.

\section{$5 \quad$ LEP Centre-of-Mass Energy measurement}

The LEP centre-of-mass energy is determined for each of the 181, 182, 183 and $184 \mathrm{GeV}$ datasets. The four values are combined taking into account the relative luminosity of each 
dataset. A Monte Carlo reweighting procedure [14] is applied to find the value of $\mathrm{E}_{C M}$ which best fits the reconstructed $x$ distribution. Selected Monte Carlo (KORALZ) signal events from the large sample at a reference energy of $182.675 \mathrm{GeV}$ are reweighted using the ratio of the differential production cross-sections [15]

$$
w_{i}\left(E_{C M}\right)=\frac{\frac{d \sigma}{d x}\left(x_{\text {true }}^{i}, E_{C M}\right)}{\frac{d \sigma}{d x}\left(x_{\text {true }}^{i}, E_{C M}^{r e f}\right)},
$$

where $x_{i}$ denotes the $x$ at which the $i$ th event has been generated. Background Monte Carlo samples are included in the fit, but they are not reweighted, i.e., their energy dependence is not taken into account. This introduces a systematic error which is estimated in section 7 .

A maximum likelihood fit is performed with fixed bins of 0.01 over the $x$ range of 0.60 0.88. The statistical error on the centre-of-mass energy is derived from the individual fits to the data distributions.

The linearity of the reweighting technique was verified using five independent Monte Carlo samples (signal and background) generated within $2 \mathrm{GeV}$ around the central nominal energy. The fitted values from these distributions have a slope $1.035 \pm 0.034$, consistent with unity, and an insignificant offset of $0.045 \pm 0.049 \mathrm{GeV}$.

\section{Results}

The number of selected events at each nominal energy, and the corresponding number of expected events are summarized in Table 2. The uncertainty on the number of expected events comes from three sources: the error on the integrated luminosity measurement, the statistical error on the estimation of the efficiencies and the theoretical uncertainty on the cross-sections.

\begin{tabular}{|c|c|c|c|}
\hline $\begin{array}{c}\text { Nominal } \\
\text { dataset }\end{array}$ & $\begin{array}{c}\text { Data } \\
\text { events }\end{array}$ & $\begin{array}{c}\text { Expected } \\
\text { events }\end{array}$ & $\begin{array}{c}\text { Integrated } \\
\text { Luminosity }\left(\mathrm{pb}^{-1}\right)\end{array}$ \\
\hline 181 & 11 & $8 \pm 1$ & $0.166 \pm 0.006$ \\
\hline 182 & 187 & $173 \pm 2$ & $3.924 \pm 0.029$ \\
\hline 183 & 2293 & $2191 \pm 12$ & $50.795 \pm 0.105$ \\
\hline 184 & 76 & $84 \pm 1$ & $1.927 \pm 0.021$ \\
\hline
\end{tabular}

Table 2: Number of events found in each energy dataset after the selection cuts, as well as the corresponding number of expected events and integrated luminosity .

The LEP centre-of-mass energy values and the statistical errors obtained from fits to the individual datasets are summarized in Table 3, as well as the weighted average according to the integrated luminosity of each dataset. The last column gives the value obtained by the LEP energy calibration group.

Figure 1 shows the reconstructed $x$ distribution for the selected events in each dataset, superimposed with the Monte Carlo reweighted expectation using the LEP centre-of-mass energy which best fits the data. 


\begin{tabular}{|c|c|c|c|c|}
\hline $\begin{array}{c}\text { Nominal } \\
\text { energy }\end{array}$ & $\begin{array}{c}\mathrm{E}_{C M}(\mathrm{fit}) \\
(\mathrm{GeV})\end{array}$ & $\begin{array}{c}-\Delta \mathrm{E}_{C M} \\
(\mathrm{GeV})\end{array}$ & $\begin{array}{c}+\Delta \mathrm{E}_{C M} \\
(\mathrm{GeV})\end{array}$ & $\begin{array}{c}\mathrm{E}_{C M}(\mathrm{LEP}) \\
(\mathrm{GeV})\end{array}$ \\
\hline 181 & 181.19 & -2.45 & +2.82 & $180.826 \pm 0.050$ \\
\hline 182 & 181.73 & -0.63 & +0.68 & $181.708 \pm 0.050$ \\
\hline 183 & 182.56 & -0.22 & +0.19 & $182.691 \pm 0.050$ \\
\hline 184 & 182.56 & -0.99 & +1.11 & $183.801 \pm 0.050$ \\
\hline \hline Combined & 182.50 & -0.20 & +0.18 & $182.652 \pm 0.050$ \\
\hline
\end{tabular}

Table 3: Fitted LEP centre-of-mass energies and corresponding statistical errors for each dataset. The last column gives the LEP centre-of-mass energies established by the LEP energy calibration group for the corresponding dataset.
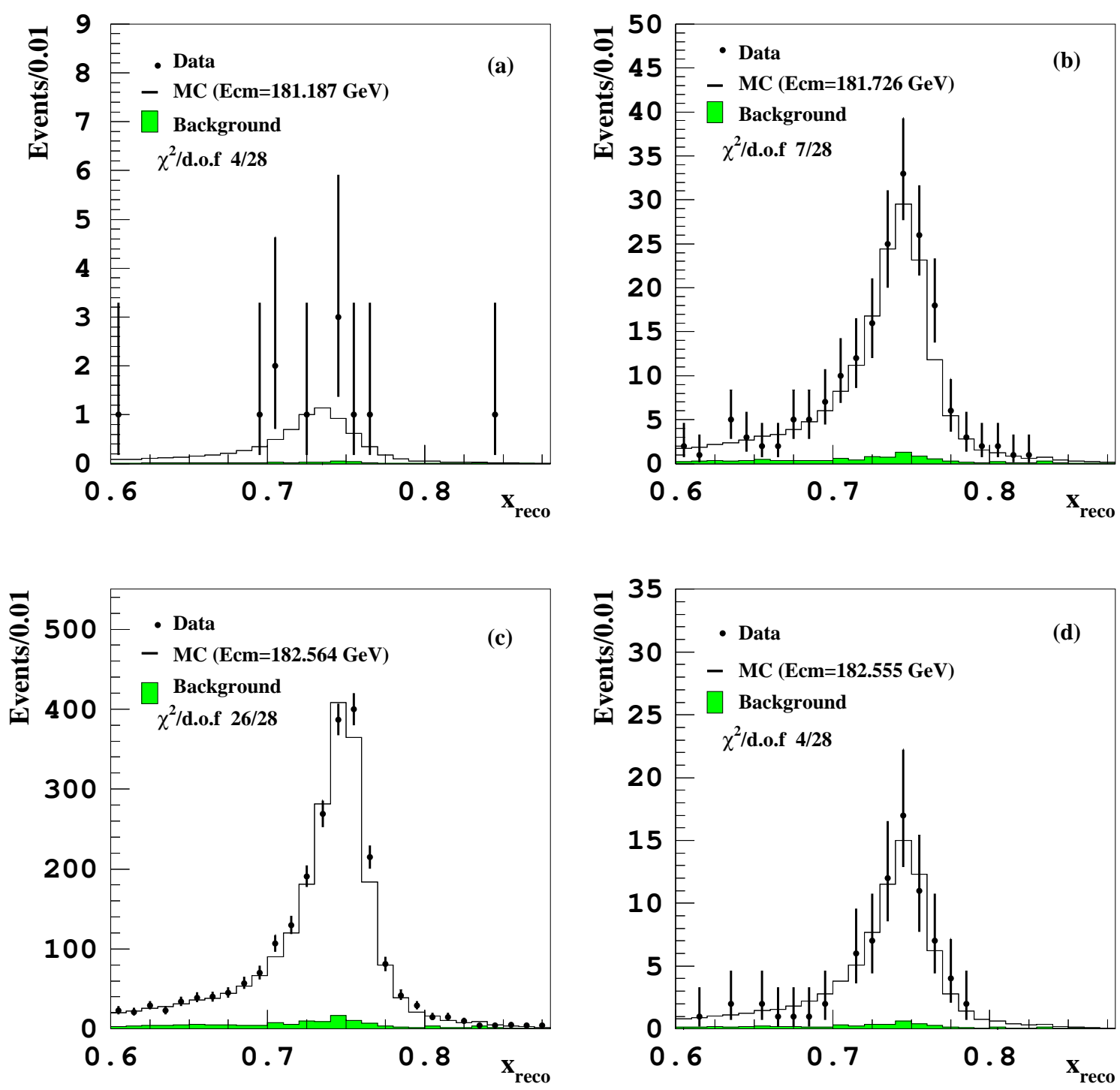

Figure 1: Reconstructed $\mathrm{x}$ distribution for selected data (dots) and background (shaded area) for each energy dataset: 181 (a), 182 (b), 183 (c) and $184 \mathrm{GeV}$ (d). The open histogram shows the results of the fit 


\section{Systematic uncertainties}

Table 1 summarizes all the systematic errors discussed below.

\begin{tabular}{|c|c|}
\hline Source & $\Delta \mathrm{E}_{C M}(\mathrm{MeV})$ \\
\hline \hline Calorimeter calibrations & 44 \\
\hline Jet energy calibration & 47 \\
\hline Jet angle calibration & 20 \\
\hline MC statistics & 24 \\
\hline ISR & 21 \\
\hline ISR-FSR interference & 10 \\
\hline Background contamination & 32 \\
\hline \hline Total & 82 \\
\hline
\end{tabular}

Table 4: Summary of the systematic errors on the LEP centre-of-mass energy measurement.

\section{Calorimeter calibrations}

During the 1997 data taking the uncertainties on the absolute calibration scale of ECAL and HCAL energies were estimated to be $\pm 0.9 \%$ and $\pm 2 \%$, respectively. Maximum shifts of $26 \mathrm{MeV}$ and $36 \mathrm{MeV}$ were observed when applying the fitting procedure to the $\mathrm{MC}$ sample after variation of ECAL and HCAL energies in both directions by the corresponding amount.

\section{Jet energy calibration}

$Z$ peak data are used to map the response of the detector to hadronic jets as a function of their polar angle. The observed difference in the energy scale of jets between data and Monte Carlo is parametrised as a function of $|\cos \theta|$, where $\theta$ is the angle between the jet direction and the beam axis. To estimate the systematic error, two modified parametrisations are derived which correspond to $\pm 1 \sigma$ uncertainty on the discrepancy. The largest shift observed when these modified parametrisations are used to correct the jet energies amounts to $47 \mathrm{MeV}$ and is taken as the systematic error.

\section{Jet angle calibration}

$Z$ peak data have been used as well to study the two jets acollinearity as a function of $|\cos \theta|$. The observed difference between data and Monte Carlo is used to estimate this systematic error. A $20 \mathrm{MeV}$ shift is observed when applying or not this correction and is quoted as the systematic error.

\section{Initial State Radiation}

ISR is described in KORALZ up to second order in the leading-log approximation $\left(\mathrm{O}\left(\alpha^{2} L^{2}\right)\right)$, in the YFS [16] style. The effect of the missing higher order terms is studied by degrading KORALZ to $\mathrm{O}\left(\alpha^{1} L^{1}\right)$. A $21 \mathrm{MeV}$ shift is observed and is quoted as an upper limit of the systematic error.

\section{ISR-FSR interference}

No existing Monte Carlo generator is able to give a good representation of initial-final state interference for quark final states. An evaluation of the order of magnitude of the effect has been performed using ZFITTER [17]. The differential cross-section $d \sigma / d x$ has 
been computed with ZFITTER, switching on and off the ISR-FSR interference, including only the polar angle region $|\cos (\theta)|<0.95$, at the energy corresponding to the fitted value in the data. In both cases these differential cross-sections are integrated in $x$ bins of 0.01 over the $x$ range of $0.60-0.88$, as for the energy measurement. The shift observed in the $Z$ resonance peak translates into an uncertainty of $10 \mathrm{MeV}$ in the determination of the LEP centre-of-mass energy.

\section{Background contamination}

The expected background level after selection cuts is $6 \%$ and the uncertainty on the contribution of the different subprocesses will affect its shape which may also depend on the centre-of-mass energy. Since the background is not reweighted, this introduces an additional source of systematic error both from the background shape and its normalization.

Background shape. The normalization uncertainty is estimated independently for each background process.

- The two-photon background, simulated with PHOT02 and PHOJET, is normalized to the data in the low visible mass region, $\mathrm{M}_{v i s}<50 \mathrm{GeV} / c^{2}$; the difference between the expected and normalized cross-sections is used to estimate the uncertainty of the process, as in [10].

- For the $W W, Z Z$ and $Z$ ee processes the uncertainties are computed from the relative variation of their respective total production cross-sections from the generated energy $(183 \mathrm{GeV})$ down to the fitted energy from the data. This is calculated using GENTLE [18] for the $W W$ process and PYTHIA for the $Z Z$ and $Z e e$ process.

Each background process is varied according to its uncertainty keeping constant the overall background level. The largest shift observed in each case is quoted in Table 5, leading to a total systematic error of $8 \mathrm{MeV}$.

\begin{tabular}{|c|c|}
\hline Process & Deviation $(\mathrm{MeV})$ \\
\hline$W W$ & 4 \\
\hline$Z Z$ & 2 \\
\hline$\gamma \gamma$ & 6 \\
\hline$Z e e$ & 3 \\
\hline \hline Total & 8 \\
\hline
\end{tabular}

Table 5: Systematic error on the centre-of-mass energy due to background shape uncertainties.

Total normalization. The systematic error coming from the uncertainty in the total background normalization is estimated by varying all the background processes up and down, according to their respective uncertainties. The largest shift observed is $31 \mathrm{MeV}$ and is quoted as the systematic error. 


\section{Summary and conclusions}

The LEP centre-of-mass energy can be determined from the kinematic reconstruction of $q \bar{q} \gamma$ events with a $q \bar{q}$ invariant mass around the $Z$ mass. The average LEP centre-of-mass energy at ALEPH for the high energy run of 1997 is measured to be

$$
E_{C M}=182.50 \pm 0.19 \text { (stat.) } \pm 0.08 \text { (syst) } \mathrm{GeV}
$$

This result is consistent with the estimate from the LEP energy working group [2] $E_{C M}=182.652 \pm 0.050 \mathrm{GeV}$.

With the expected increase in statistics at LEP2 and with refined experimental techniques akin to those used for the $W$ mass determination [4], the method described here should provide an alternative measurement of the LEP centre-of-mass energy, with competitive accuracy. Already, if only this evaluation of the LEP centre-of-mass-energy were used in the $W$ mass determination, the systematic error on $M_{W}$ coming from the precision on the beam energy scale would be $90 \mathrm{MeV},\left(\Delta M_{W} / M_{W}=\Delta E_{C M} / E_{C M}\right)$, which is smaller than the experimental error of $139 \mathrm{MeV}$ on $M_{W}$ from the same data sample [4].

\section{Acknowledgements}

It is a pleasure to congratulate our colleagues from the CERN accelerator divisions for the successful operation of LEP2. We are indebted to the engineers and technicians in all our institutions for their contributions to the excellent performance of ALEPH. Those of us from non-member countries thank CERN for its hospitality and support.

\section{References}

[1] LEP energy working group. R. Assman et al., Calibration of centre-of-mass energies at LEP1 for precise measurements of Z properties, Eur. Phys. J. C 6 (1999) 187

[2] LEP energy working group. R. Assman et al., Evaluation of the LEP centre-of-mass energy above the $W$-pair production threshold, CERN-EP/98-191, CERN-SL/98073, submitted to European Physical Journal C.

[3] http://www.cern.ch/LEPECAL/spectro/Welcome.htm]

[4] ALEPH Collaboration, Measurement of the $W$ mass in $e^{+} e^{-}$collisions at $183 \mathrm{GeV}$, Phys. Lett. B 453 (1999) 121.

[5] ALEPH Collaboration, ALEPH: A detector for electron-positron annihilations at LEP, Nucl. Inst. and Meth. A 294, 121 (1990)

[6] ALEPH Collaboration, Performance of the ALEPH detector at LEP, Nucl. Inst. and Meth. A 360, 481 (1995)

[7] S. Jadach, B.F.L. Ward and Z. Wạs, Comput. Phys. Commun. 79 (1994) 503. 
[8] T. Sjöstrand, Comput. Phys. Commun. 82 (1994) 74.

[9] M. Skrzypek, S. Jadach, W. Placzek and Z. Wạs, Comp. Phys. Commun. 94 (1996) 216.

[10] ALEPH Collaboration, Study of Fermion pair production in $e^{+} e^{-}$collisions at 130$183 \mathrm{GeV}$, CERN EP/99-042. Submitted to European Physical Journal C.

[11] J.A.M Vermaserem, Proceedings of the IV International Workshop on Gamma Gamma Interactions, eds, G. Cochard and P. Kessler (1980)

[12] R. Engel, Z. Phys. C 66 (1995) 203;

R. Engel and J. Ranft, Phys. Rev. D 54 (1996) 4144.

[13] W. Bartel et al., Z. Phys. C 33 (1986) 23.

[14] ALEPH Collaboration, Measurement of the $W$ Mass by direct Reconstruction in $e^{+} e^{-}$Collisions at $172 \mathrm{GeV}$, Phys. Lett. B 422 (1998) 384.

[15] ALEPH Collaboration, Study of the muon-pair production at centre-of-mass energies from 20 to $136 \mathrm{GeV}$ with the ALEPH detector, Phys. Lett. B 399 (1997) 329.

M.Martinez, L.Garrido, R.Miquel, J.L.Harton and R.Tanaka, Z. Phys. C 49 (1991) 645 ; and

M.Martinez and F.Teubert, Z. Phys. C 65 (1995) 267-276.

[16] D.R. Yennie, S.C. Frautschi and H. Suura, Annuals of Phys. 13 (1961) 379;

S. Jadach and B.F.L. Ward, TPJU 15/88 (1988).

[17] D.Bardin et al., Z. Phys. C 44 (1989) 493;

Comput. Phys. Commun. 59 (1990) 303;

Nucl. Phys. B 351 (1991) 1;

Phys. Lett. B 255 (1991) 290 and

CERN-TH/6443-92 (May 1992).

[18] D. Bardin et al. ; DESY 96-233, hep-ph/9612409. 